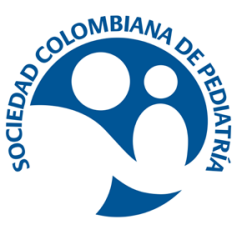

\title{
Original
}

\section{Concordancia entre el puntaje de la escala modificada de Wood y el lugar de manejo de los pacientes con bronquiolitis aguda*}

\author{
Johanna Ramírez-Villamizara, Laura Jimena Hernández-Corredor ${ }^{b}$, Jorge Luis Córdoba- \\ Gravinic, José Luis Junco-González ${ }^{d}$.
}

a Médico especialista en pediatra, Universidad Militar Nueva Granada. Especialista en epidemiología, Universidad del Rosario. Bogotá, Colombia. Correo electrónico: Ramirezv.johanna@gmail.com

b Médico, residente de pediatría, Universidad de los Andes, Hospitalaria servicio de pediatría, Instituto Roosevelt. Correo electrónico: Lj. hernandez106@gmail.com

c Médico especialista en pediatra, Universidad de la Sabana. Correo electrónico: Jl.cordoba90@gmail.com

d Pediatra Intensivista, Instituto de Ortopedia Infantil Roosevelt. Correo electrónico: jjunco@ioir.org.co, josejuncog@gmail.com

*Institución en la que se realizó el trabajo Instituto Roosevelt, Dirección de educación e investigación

INFORMACIÓN DEL ARTÍCULO

Historia del artículo:

Recibido el 14 de febrero de 2020

Aceptado el 19 de octubre de 2020

Palabras clave:

Bronquiolitis

índice de severidad de la enfermedad

hospitalización

cuidados críticos
R E S U M E N

Introducción: La escala de severidad clínica de asma modificada de Wood (M-WCAS), validada en Colombia, es una herramienta útil para medir la severidad en bronquiolitis aguda. La escala podría evitar la variabilidad interobservador y así apoyar la decisión acerca del lugar de manejo de los pacientes (ambulatorio, hospitalización, unidad de cuidado intensivo pediátrico). Objetivo: Estimar la concordancia que existe entre la severidad de la bronquiolitis aguda medida la escala M-WCAS en el primer momento de atención, el lugar de manejo y los días de estancia hospitalaria de los pacientes con bronquiolitis aguda en un centro de referencia pediátrico. Métodos: Estudio de concordancia, retrospectivo. Se revisaron 364 historias clínicas de pacientes con bronquiolitis aguda y se calculó el puntaje de la escala M-WCAS para cada paciente. Se estimó la concordancia entre el puntaje de la escala al ingreso hospitalario, con el lugar de manejo (ambulatorio, piso, unidad de cuidado intensivo pediátrico) y los días de estancia de los pacientes con manejo intrahospitalario. Resultados: índice de kappa de -0,51 entre la severidad clínica por la escala M-WCAS con el lugar de manejo y un coeficiente de concordancia de Lin de 0.03 entre el puntaje M-WCAS y los días de estancia hospitalaria. Conclusiones: No se evidenció concordancia entre la severidad clínica de los pacientes con bronquiolitis aguda medida por la escala M-WCAS con el lugar de manejo. Se encontró una concordancia leve entre el puntaje de la escala M-WCAS y los días de estancia hospitalaria.

\footnotetext{
*Autor para correspondencia. Johanna Ramírez-Villamizar
} Correo electrónico: Ramirezv.johanna@gmail.com

Como Citar: Ramírez-Villamizar J, Hernández-Corredor LJ, Córdoba-Gravini JL, Junco-González JL. Concordancia entre el puntaje de la escala modificada de Wood y el lugar de manejo de los pacientes con bronquiolitis aguda. Pediatr. 2020;53(2):42-48. 


\title{
Wood's modified scale concordance with management location of patients with acute bronchiolitis
}

\author{
A B S T R A C T
}

Keywords:

Bronchiolitis

illness Severity Index

hospitalization

critical Care Unit.

\begin{abstract}
Introduction: Modified Wood's clinical asthma severity scale (M-WCAS), a validated scale in Colombia, is a useful tool to measure severity in acute bronchiolitis. The scale could prevent interobserver variability, and support better patients' management location (ambulatory, hospitalization, intensive care unit). Objective: To estimate the concordance between the severity of acute bronchiolitis measured by M-WCAS, management location, and hospital stay days of patients with acute bronchiolitis in a pediatric referral center. Methods: In a retrospective concordance study, 364 medical records of patients with acute bronchiolitis were reviewed. Researchers calculated the M-WCAS score. Concordance was estimated between the scale score at admission, management location, and hospital stay days of patients who required in-hospital treatment. Results: We found a kappa index of -0.51 between clinical severity by M-WCAS scale and management location. A Lin concordance coefficient of 0.03 between M-WCAS score and hospital stay days. Conclusions: We found no correlation between the clinical severity of bronchiolitis measured by the M-WCAS scale with the patient's management location. We identified a slight concordance between the M-WCAS score, and hospital stay days
\end{abstract}

\section{Introducción}

La bronquiolitis es una infección respiratoria aguda definida como el primer episodio de sibilancias o roncus precedido de un cuadro gripal que afecta a los menores de 2 años, es de etiología viral, predominando en época de lluvias $(1,2,3)$. Es una patología de relevancia social, según la guía de práctica clínica del Ministerio de Salud del año 2014, usada para la evaluación del riesgo y manejo inicial de la bronquiolitis en niñas y niños menores de 2 años, esta patología ocupa el sexto lugar de todas las consultas, el cuarto lugar de las causas de hospitalizaciones y el séptimo lugar de todas las causas de muerte en Colombia (4). El curso clínico de la bronquiolitis es dinámico y varía desde un evento leve transitorio hasta dificultad respiratoria severa $(2,5,6)$. El diagnóstico y clasificación de la severidad de la bronquiolitis se basan en la historia clínica y el examen físico $(2,5)$. Los exámenes de laboratorio y toma de radiografía no se recomiendan de manera rutinaria (2). Los hallazgos del examen físico, que señalan severidad, son la taquipnea, el uso de músculos accesorios y la saturación de oxígeno baja (2). En cuanto a los factores de riesgo identificados que predicen evolución a bronquiolitis severa, descritos en la literatura, estos son la edad entre 6 semanas a 12 meses de vida, prematuridad, enfermedades cardiopulmonares, inmunodeficiencias, desordenes neuromusculares y la desnutrición $(5,6,7,8,9,10)$.

Según la literatura, existe amplia variabilidad en el ámbito clínico, del uso de los predictores de hospitalización de los pacientes con diagnóstico de bronquiolitis $(5,11,12)$ siendo importante clasificar adecuadamente, el lugar de manejo para cada paciente, según sea ambulatorio, hospitalización o en la unidad de cuidado intensivo pediátrico (UCIP), de acuerdo con la severidad del cuadro, antecedentes médicos y rasgos sociodemográficos $(1,10)$.

De esta manera se podría disminuir la morbilidad y mortalidad de los pacientes, optimizando los recursos sanitarios. La escala M-WCAS es un método reproducible y válido para medir la severidad de la bronquiolitis aguda y que podría ser una herramienta de apoyo a la hora de tomar decisiones en cuanto al lugar de manejo de estos pacientes $(12,13,14,15)$. Además, la escala fue validada en Colombia en el año 2013 (16).

Esta investigación tiene como objetivo, estimar la concordancia entre el puntaje de la escala de severidad clínica de asma modificada de Wood (M-WCAS) en pacientes afectados por bronquiolitis, con el lugar de manejo (ambulatorio, piso, UCIP) y los días de estancia hospitalaria en un centro de referencia pediátrico.

\section{Métodos}

Estudio descriptivo, retrospectivo, de concordancia. La fuente de información fueron las historias clínicas de los pacientes menores de 2 años con diagnóstico de bronquiolitis aguda atendidos en el Instituto de Ortopedia Infantil Roosevelt.

El tamaño de muestra se estimó a partir de estudios internacionales previos, en donde la prevalencia de hospitalización por bronquiolitis fue calculada en $4 \%$ (1) y teniendo en cuenta la población de menores de dos años en la ciudad de Bogotá en el año 2015, estimada en 363038 niños según el calendario estadístico 2015 de la Secretaria Distrital de Planeación de Bogotá. No se encontraron estudios similares que reportaran la magnitud de concordancia entre el lugar de 
manejo y el puntaje de la escala M-WCAS, por lo que se realizó una prueba piloto, asumiendo una frecuencia esperada del $50 \%$, para estimar el cálculo de tamaño de muestra.

Se tomó como marco muestral la base de datos de las historias clínicas de los pacientes menores de 24 meses de edad hospitalizados en el Instituto Roosevelt, durante enero del año 2008 y enero del 2015 con diagnósticos CIE-10 de: bronquiolitis aguda no especificada, bronquiolitis aguda debida a virus sincitial respiratorio y bronquiolitis aguda debida a otros microorganismos especificados. El total de historias clínicas con la búsqueda inicial fue de 5080 . Uno de los investigadores depuró, de la base de datos, aquellas historias con antecedente CIE-10 de Bronquiolitis, es decir cuyo motivo de hospitalización era otro código CIE-10 diferente, obteniéndose un total de 4957 historias.

Se estimó el tamaño de la muestra con Statcalc del programa Epi info $7^{\mathrm{TM}}$ reduciendo el tamaño poblacional a 14521 menores de 2 años y considerando una confiabilidad del 95\%. El tamaño de la muestra calculado fue de 364 historias clínicas, las cuales fueron seleccionadas con una tabla de números aleatorios basada en Excel ${ }^{\circledR}$.

Los tres investigadores revisaron las historias clínicas, verificaron que cumplieran con los criterios de definición de bronquiolitis aguda y calcularon el puntaje de la escala M-WCAS con los datos de frecuencia respiratoria, frecuencia cardiaca, presencia de cianosis, sibilancias, tirajes y descripción de la ventilación, registrados el día de la atención inicial para cada paciente.

Se calculó el estadístico de kappa ponderado, para la evaluación de la concordancia entre la variable ordinal: puntaje de la escala M-WCAS y la variable nominal: lugar de manejo de los pacientes, con sus tres categorías (ambulatorio, hospitalización en pisos y hospitalización en UCIP). La interpretación del valor del estadístico de Kappa se realizó mediante la categorización de valores de Landis y Koch $(17,18)$. Para el cálculo del kappa ponderado se realizó inicialmente un control por estratificación de los pacientes según si tenían o no antecedentes patológicos que predecían la evolución a bronquiolitis severa, descritos en la literatura, tales como: edad entre 6 semanas a 12 meses de vida, prematuridad, enfermedades cardiopulmonares, inmunodeficiencias, desordenes neuromusculares y nutricionales $(5,6,7,8,9,10)$.

Se utilizó el coeficiente de concordancia de Lin para la evaluación de la concordancia entre la variable puntaje de la escala M-WCAS y la variable cuantitativa continua: días de estancia hospitalaria. Se utilizó el programa estadístico SPSS ${ }^{\circledR}$ bajo licencia de la Universidad del Rosario, para procesar los datos.

Según la Resolución 8430, este estudio se considera una investigación sin riesgo. Se emplearon técnicas y métodos de investigación documental retrospectiva que no contemplaron la realización de ninguna intervención o modificación intencionada de las variables biológicas, fisiológicas, psicológicas o sociales de los individuos que participaron en el estudio. El protocolo de investigación fue aprobado por el comité de ética de la investigación del Instituto Roosevelt.

\section{Resultados}

La mediana de la edad de los pacientes con bronquiolitis aguda fue de un mes, con una desviación estándar de 5.02 meses, la edad mínima fue de 0 meses y la máxima de 24 meses. Los meses de atención por bronquiolitis aguda más frecuentes fueron marzo y abril (Tabla 1). El $82.7 \%$ de los pacientes tenían el esquema del programa ampliado de inmunizaciones (PAI) al día. El $76.7 \%$ recibían lactancia materna. En el $35.2 \%$ de los pacientes atendidos, se aisló, en el panel respiratorio, virus sincitial respiratorio como agente causal de bronquiolitis (Ver gráfica 1).

\section{Tabla 1. Variables sociodemográficas}

\begin{tabular}{|c|c|c|}
\hline \multirow{2}{*}{ Variable } & Frecuencia & Porcentaje \\
\hline & $\mathrm{n}: 364$ & $\%$ \\
\hline \multicolumn{3}{|l|}{ Género } \\
\hline Masculino & 192 & 52.7 \\
\hline Femenino & 172 & 47.3 \\
\hline \multicolumn{3}{|c|}{ Mes de atención } \\
\hline Enero & 25 & 6.9 \\
\hline Febrero & 16 & 4.4 \\
\hline Marzo & 45 & 12.4 \\
\hline Abril & 45 & 12.4 \\
\hline Mayo & 33 & 9.1 \\
\hline Junio & 35 & 9.6 \\
\hline Julio & 20 & 5.5 \\
\hline Agosto & 24 & 6.6 \\
\hline Septiembre & 29 & 8 \\
\hline Octubre & 32 & 8.8 \\
\hline Noviembre & 28 & 7.7 \\
\hline Diciembre & 32 & 8.8 \\
\hline \multicolumn{3}{|c|}{ Zona de residencia } \\
\hline Rural & 7 & 1.9 \\
\hline Sin dato & 1 & 0.3 \\
\hline Urbana & 356 & 97.8 \\
\hline \multicolumn{3}{|c|}{ Régimen de seguridad social } \\
\hline Contributivo & 355 & 97.5 \\
\hline Subsidiado & 7 & 1.9 \\
\hline Otros & 2 & 0.5 \\
\hline \multicolumn{3}{|c|}{ Lactancia materna } \\
\hline $\mathrm{Si}$ & 279 & 76.7 \\
\hline No & 19 & 5.2 \\
\hline \multicolumn{3}{|l|}{ Vacunación } \\
\hline $\mathrm{Si}$ & 301 & 82.7 \\
\hline No & 14 & 3.8 \\
\hline Sin dato & 49 & 13.5 \\
\hline \multicolumn{3}{|l|}{ Reingreso } \\
\hline $\mathrm{Si}$ & 17 & 4.7 \\
\hline \multicolumn{3}{|c|}{ Virus sincitial respiratorio } \\
\hline Negativo & 185 & 50.8 \\
\hline Positivo & 128 & 35.2 \\
\hline Sin Dato & 51 & 14 \\
\hline
\end{tabular}




\section{Distribución de virus sincitial respiratorio por edad}

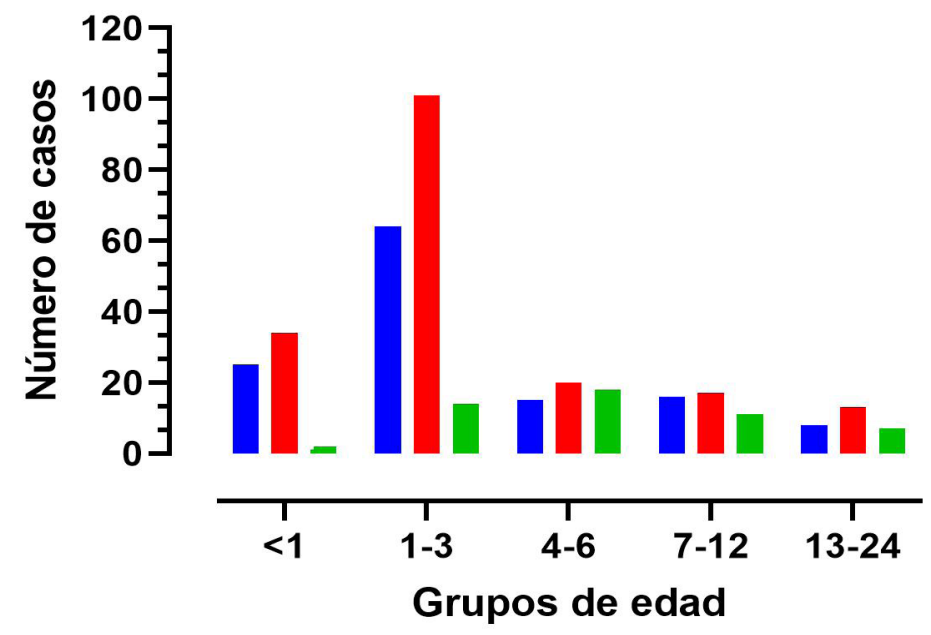

VSR+

VSR-

Sin dato

\section{Grafica 1.}

De las 364 historias clínicas revisadas a las cuales se les aplicó la escala M-WCAS, según los datos registrados en la historia clínica al momento del ingreso, 322 (88 \%) obtuvieron un puntaje entre 0 y 3 (levemente enfermo), 19 (5\%) entre 4 y 6 (moderadamente enfermo) y $2(1 \%$ un puntaje mayor de 6 (severamente enfermo), en 21 historias clínicas no se encontraron los datos completos para el diligenciamiento de la escala (Ver gráfica 2). Según grupos de edad hubo 1 menor de 1 mes de edad y otro entre 13 y 24 meses de edad severamente enfermos, la distribución de severidad por grupos de edad se aprecia en la gráfica 3.

Un total de 115 pacientes fueron manejados de manera ambulatoria (32\%), 216 pacientes se hospitalizaron en piso (59\%) y 33 pacientes ingresaron a la unidad de cuidado intensivo pediátrico (9\%). La mediana de estancia hospitalaria fue de 4 días con un límite inferior de 1 día y superior de 23 días. La estancia de los pacientes severamente enfermos fue entre 3 y 4 días. La distribución de días de estancia por severidad se aprecia en el gráfico 4 . El $14 \%$ de los pacientes de la muestra tenían antecedentes patológicos descritos en la literatura, como factores de riesgo que predicen evolución a bronquiolitis severa como prematuridad, enfermedades

\section{Clasificación de la severidad según puntaje de M-WCAS}

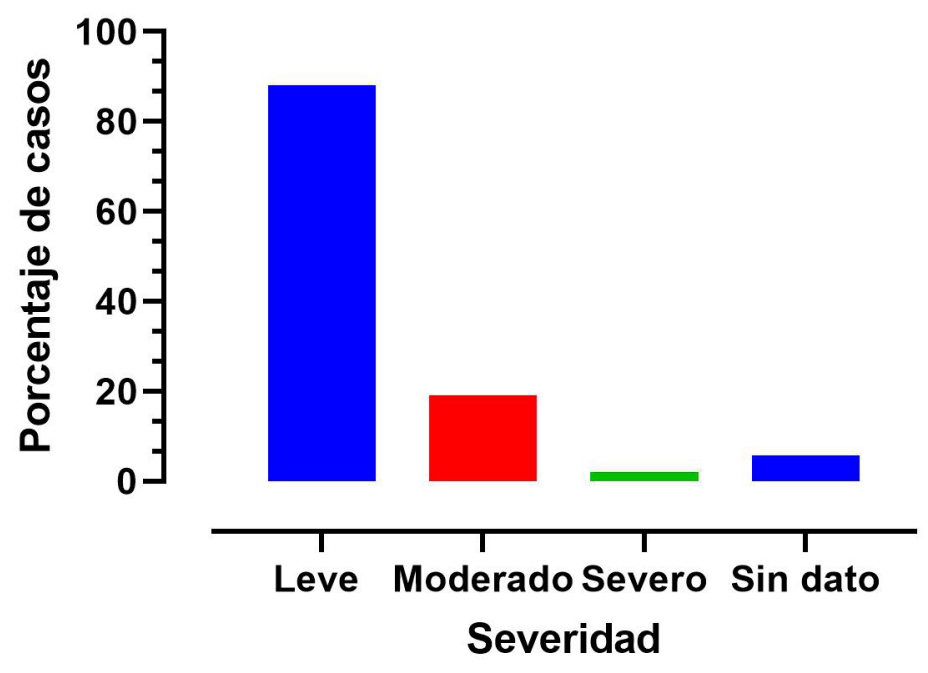

Grafica 2.

\section{Severidad}




\section{Distribución de severidad por grupo de edad}

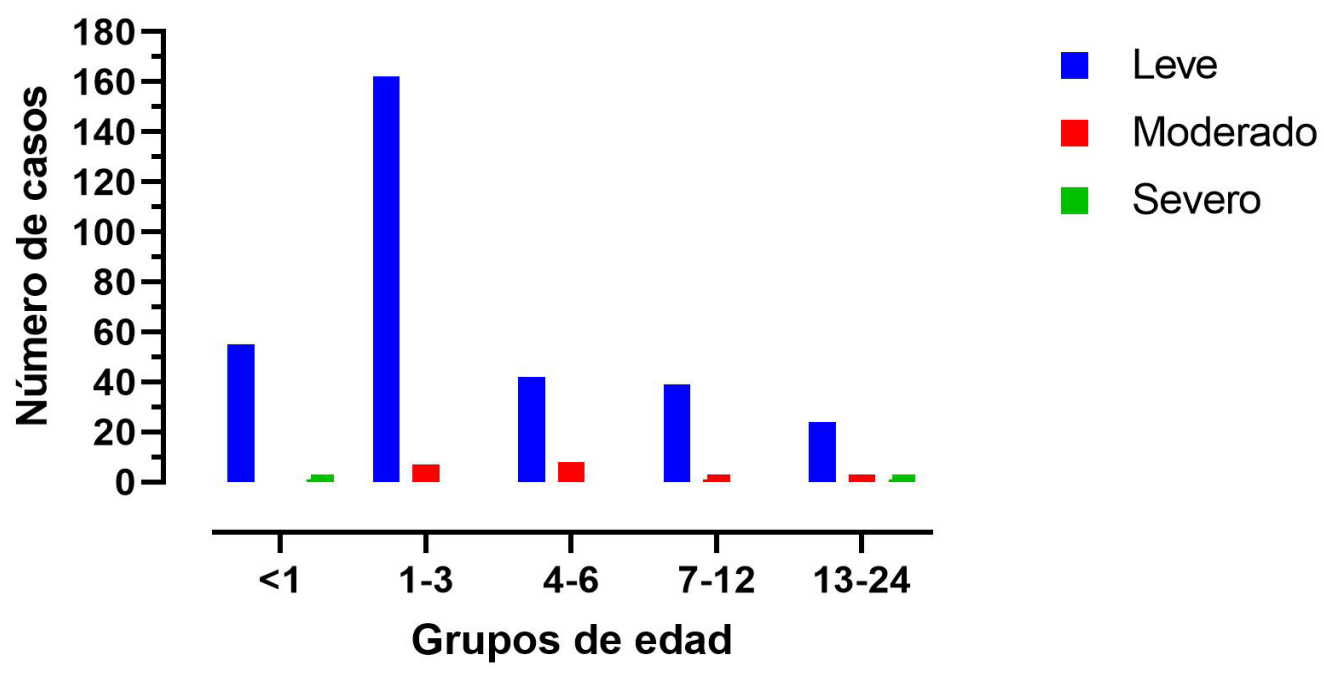

Grafica 3.

\begin{tabular}{lll}
\hline Tabla 2. Frecuencia de los antecedentes patológicos & \\
\hline Antecedente & Frecuencia & Porcentaje \\
\hline Ano imperforado & 1 & 0.3 \\
\hline Aspiración de meconio & 2 & 0.5 \\
\hline Broncoaspiración & 1 & 0.3 \\
\hline CIA* ostium secundum & 1 & 0.3 \\
\hline Cierre de ductus quirúrgico, prematurez & 1 & 0.3 \\
\hline Coartación de aorta, arteria subclavia aberrante & 1 & 0.3 \\
\hline Desnutrición & 1 & 0.3 \\
\hline Epilepsia & 1 & 0.3 \\
\hline Hipertensión pulmonar & 2 & 0.5 \\
\hline Mielomeningocele & 1 & 0.3 \\
\hline Obesidad & 1 & 0.3 \\
\hline Prematurez & 30 & 8.2 \\
\hline Displasia broncopulmonar & 6 & 1.6 \\
\hline RCIU** & 2 & 0.5 \\
\hline Sin Antecedentes & 313 & 86 \\
\hline Total & 364 & 100 \\
\hline
\end{tabular}

*CIA: comunicación interauricular.

${ }^{*}$ RCIU: retraso del crecimiento intrauterino.

cardiopulmonares, desórdenes neurológicos y nutricionales, tabla 2.

Para examinar la concordancia entre el puntaje de la escala de asma modificada de Wood (M-WCAS) calculada al momento de la atención médica inicial y el lugar de manejo de los pacientes con bronquiolitis aguda (ambulatorio, piso, UCIP), se utilizó el método estadístico de Kappa, con un índice de kappa obtenido de $\mathrm{K}$ : - 0,51, lo cual indica discordancia entre ambos parámetros $(17,18)$. La estimación del coeficiente de concordancia de Lin entre las variables puntaje de la escala M-WCAS aplicada a los pacientes en el momento de la atención inicial en un hospital de referencia pediátrica y los días de estancia hospitalaria fue de 0.03, [IC-95 \% 0,011-0,064], indicando concordancia leve entre dichas variables. 


\section{Discusión}

Se ha observado recientemente que las hospitalizaciones por bronquiolitis aguda vienen en aumento en los últimos años, pero a pesar de ello la tasa de mortalidad por esta patología se mantiene constante (5). Lo anterior se ha visto aparentemente relacionado con la introducción de la pulso-oximetría desde el año 1980 (11) ya que desde entonces este parámetro se considera como el quinto signo vital y es un factor determinante a la hora de tomar la decisión de hospitalizar al paciente, en ocasiones llega a ser la única justificación de hospitalización $(19,20,21)$, la cual puede tener lugar en casa con oxígeno suplementario domiciliario, actualmente esta indicación ocupa uno de los criterios más comunes de hospitalización en un niño con bronquiolitis aguda seguido del requerimiento de líquidos endovenosos $(7,20,22)$. Al revisar las historias clínicas de manera retrospectiva, encontramos que la mayoría de los pacientes fueron clasificados como levemente enfermos (88\%), sin embargo, solo el 32 \% fueron manejados de manera ambulatoria y adicionalmente la escala empleada M-WCAS para evaluar la severidad de la bronquiolitis aguda en nuestro estudio no incluye la variable de saturación de oxígeno ni tampoco toma en cuenta antecedentes patológicos que han demostrado predecir el riesgo de complicaciones $(5,6,7,8,9,10)$ y por lo tanto ameritan seguimiento estrecho por riesgo de deterioro clínico.

Consideramos como otra limitación de nuestro estudio la metodología utilizada, ya que al aplicar la escala retrospectivamente no garantizamos que esta sea utilizada en condiciones clínicas similares, ni permite una aplicación simultánea al momento de la atención inicial, el evaluador no es el mismo profesional que atendió al paciente lo cual limita la rigurosidad y calidad de implementación de la escala, adicionalmente la calidad de la historia clínica puede afectar la evaluación. En 21 pacientes no se pudo calcular el puntaje por no tener todos los datos registrados en la historia clínica de ingreso. Por otro lado, no hubo una distribución similar del número de pacientes en las tres categorías de severidad de la escala, para examinar la concordancia en los paciente moderada y severamente enfermos con el lugar de manejo (ambulatorio, piso, UCIP) y los días de estancia hospitalaria.

Se requieren estudios prospectivos para evaluar la concordancia entre la escala de severidad de asma clínica modificada de Wood, la conducta médica en cuanto al lugar de manejo y los días de estancia. Como fortaleza de nuestro estudio resaltamos el tamaño de muestra la cual es representativa de la población a estudio y es probabilística.

\section{Conclusiones}

Encontramos en nuestro estudio discordancia entre el puntaje de la escala M-WCAS en los pacientes con bronquiolitis aguda en el primer momento de atención con el lugar de manejo de estos pacientes (ambulatorio, piso, UCIP) y una concordancia leve entre el puntaje de la escala M-WCAS y los días de estancia hospitalaria.
Agradecimientos al Instituto Roosevelt y al Departamento de Epidemiología de la Universidad del Rosario.

\section{R E F E R E N C I A S}

1. Piñero FJ, Alfayate MS, Menasalvas RA, et al. Características epidemiológicas, clínicas y terapéuticas de lactantes hospitalizados por bronquiolitis. AnPediatr. 2012; 77(6):391-6.

2. Ricci V, Delgado Nunes V, Murphy MS, Cunningham S. Bronchiolitis in children: summary of NICE guidance. Bmj. 2015; 350(14): 2305-2305.

3. Ralston $\mathrm{S}$, Lieberthal A, Meissner H, et al. American Academy of Pediatrics. Clinical Practice Guideline: The Diagnosis,Management, and Prevention of Bronchiolitis. Pediatrics. 2014; 134:1474-1502.

4. Ministerio de salud y protección social. Guía de práctica clínica del ministerio de salud para la evaluación del riesgo y manejo inicial de la neumonía en niñas y niños menores de 5 años y bronquiolitis en niñas y niños menores de 2 años. 2014; 42:1356.

5. Ricart S, Marcos M, Sarda M, Anton A, Muñoz-Almagro C, Pumarola T, et al. Clinical risk factors are more relevant than respiratory viruses inpredicting bronchiolitis severity. Pediatr Pulmonol. 2013; 48(5):456-63.

6. Riese J, McCulloh RJ, Koehn KL, Alverson BK. Demographic Factors Associated with Bronchiolitis Readmission. Hosp Pediatr. 2014; 4(3):147-52.

7. Yusuf S, Caviness AC, Adekunle-Ojo AO. Risk Factors for Admission in Children with Bronchiolitis From Pediatric Emergency Department Observation Unit. Pediatr Emerg Care. 2012; 28(11):1132-1135.

8. Corneli HM, Zorc JJ, Holubkov R, Bregstein JS, Brown KM, Mahajan P, et al. Bronchiolitis: clinical characteristics associated with hospitalization and length of stay. Pediatr Emerg Care. 2012; 28(2): 99-103.

9. García C, Bhore R, Soriano-Fallas A. Risk Factors for Admission in Children with Bronchiolitis From Pediatric Emergency Department Observation Unit. Pediatrics. 2010; 126(6):11321135.

10. Corneli HM, Zorc JJ, Holubkov R, Bregstein JS, Brown KM, Mahajan P, et al. Bronchiolitis: clinical characteristics associated with hospitalization and length of stay. Pediatr Emerg Care. 2012; 28(2):99-103.

11. Mallory M, Shay D, Garrett J, Bordley C. Bronchiolitis management preferences and the influence of pulse oximetry and respiratory rate on the decision to admit. Pediatrics. 2003; 111:45-51.

12. Ramos J, Cordón A, Galindo R, Urda A. Validación de una escala clínica de severidad de la bronquiolitis aguda. AnPediatr, 2014; 81(1):3-8

13. Bekhof J, Reimink P, Brand P. Systematic review: Insufficient validation of clinical scores for the assessment of acute dyspnoea in wheezing children. Pediatric Respiratory Reviews. 2014; 15: 98-112.

14. Luarte Martinez S, Rodriguez I, Astudillo P, Manterola C. Psychometric properties of scales used for grading the severity of bronchial obstruction in pediatrics: A systematic review and meta-analysis. Arch Argent Pediatr. 2017;115(3):241-248. 
15. Rodriguez C, Sossa M, Nino G. Systematic review of instruments aimed at evaluating the severity of bronchiolitis. Paediatr Respir Rev. 2018; 25:43-57.

16. Duarte-Dorado DM, Madero-Orostegui DS, Rodriguez-Martinez CE, Nino G. Validation of a scale to assess the severity of bronchiolitis in a population of hospitalized infants. J. Asthma. 2013; 50(10):1056-61.

17. Carrasco J, Jover L. Métodos estadísticos para evaluar la concordancia. Med Clin. Elsevier; 2004;122(1):28-34.

18. Cerda J, Villaroel del P. Evaluación de la concordancia interobservador en investigación pediátrica: Coeficiente de Kappa. Rev Chil Pediatr. 2008; 79 (1): 54-58.

19. McCallum G, Morris P, Wilson C, Versteegh L, Ward L, Chatfield $\mathrm{M}$, et al. Severity scoring systems: are they internally valid, reliable and predictive of oxygen use in children with acute bronchiolitis? Pediatr Pulmonol. 2013; 48(8):797-803.

20. Rodríguez D, Rodríguez-Martínez C, Cárdenas A, Quilaguy I, Mayorga L, Falla L, et al. Predictors of severity and mortality in children hospitalized with respiratory syncytial virus infection in a tropical region. Pediatr Pulmonol. 2014; 49 (3): 269-76.

21. Hervás D, Reina J, Yanez A, Del Valle JM, Figuerola J, Hervás J, Epidemiology of hospitalization for acute bronchiolitis in children: Differences between RSV and non-RSV bronchiolitis. Eur J Clin Microbiol Infect Dis. 2012; 31(8):1975-81.

22. Sala K, Moore A, Desai S, Welch K, Bhandari S, Carroll C. Factors associated with disease severity in children with bronchiolitis. 2015; 52 (3):268-272. 